\title{
Efficacy of Sofosbuvir-Daclatasvir in Treatment of Chronic Hepatitis C Virus Infection
}

\author{
Y.S.Younis ${ }^{1}$, T.E.Elazab ${ }^{1}$ and E.M.Abd El-Fattah ${ }^{2}$
}

${ }^{1}$ Hepatology, Gastroenterology and Infectious Diseases Dept., Faculty of Medicine, Benha Univ., Benha, Egypt

${ }^{2}$ Qina Fever Hospital, Ministry of Health, Qina City, Egypt.

E-Mail: E.M.Abd El-fattah22@gmail.com

\begin{abstract}
The highest prevalence of chronic hepatitis $\mathrm{C}$ virus $(\mathrm{HCV})$ was reported in Egypt. DAAs has been accessible, with a revealed 12 weeks continued virologic reaction (12w-SVR) above $95 \%$ after treatment for 12 weeks duration . was to assess adequacy and wellbeing of Sofosbuvir in addition to Daclatasvir with or without Ribavirin in Egyptian patients persistently contaminated with HCV . Study was done on 200 patients separated similarly to 2 enormous gatherings; bunch I under 65 years and gathering II over 65 years partitioned into 4 subgroups rely upon ribavirin. The first subgroup will take sofosbuvir 400mg and daclatasavir60mg (double treatment gathering), under 65 years of age (bunch Ia ). The subsequent subgroup will take sofosbuvir 400mg, daclatasvir $60 \mathrm{mg}$ and ribavirin 1000-1200 mg weight based (triple treatment gathering) under 65 years of age ( bunch $\mathrm{Ib}$ ). The third subgroup will take sofosbuvir $400 \mathrm{mg}$, daclatasvir $60 \mathrm{mg}$ (dual treatment group)more than 65 years of age( bunch IIa )and the fourth subgroup will take sofosbuvir 400mg, daclatasvir $60 \mathrm{mg}$ and ribavirin1000-1200 mg weight based(triple treatment gathering) (bunch IIb ). The current examination show that the treatment was sheltered and compelling in all gatherings with by and large reaction of $96 \%, 94 \% 92 \%$ and $94 \%$ in bunch $\mathrm{Ia}$, Ib, IIa, IIb respectively.
\end{abstract}

Keywords: Sofosbuvir, Daclatasvir, Ribavirin, HCV.

\section{Introduction}

Ongoing HCV contamination is a significant reason for constant liver sickness, cirrhosis and liver malignant growth just as a significant sign for liver transplantation [18]. Egypt has the most elevated predominance of hepatitis $\mathrm{C}$ infection (HCV) disease on the planet. The commonness of HCV viremia was assessed to be $7.3 \%$ for the year 2013 dependent on information from the 2008 Egypt Demographic and Health Survey [8]. Up to 2011, blend of week after week peginterferon- $\alpha$ (peg-IFN $\alpha$ ) and every day portions of RBV in a 24 or 48 -week course was the standard therapy for constant HCV disease [17] The presentation of legitimately acting antiviral operators (DAAs) in the treatment of HCV contaminations has changed the field. DAAs treatment currently implies that most patients can be effectively relieved with regularly diminishing lengths of all around endured regimens [12].

The dish genotypic mix of daclatasvir and sofosbuvir, with or without ribavirin, has accomplished high SVR rates in stage 2 and stage 3 examinations. Daclatasvir is an inhibitor of the HCV NS5A replication complex; sofosbuvir is a nucleotide inhibitor of the HCV NS5B polymerase . Both have ideal wellbeing profiles and are dosed once every day, with few clinically noteworthy drug-drug interaction [24].

\section{Aims of Study}

In this study, we aimed to evaluate efficacy of Sofosbuvir plus Daclatasvir with or without Ribavirin and evaluate efficacy and safety of this regemins.

\section{Subjects and methods}

3.1Studied patients

Prospective follow up study will be conducted on 200 Egyptian patients gave ongoing HCV contamination were selected the current examination.

their age ran between 18-75 and they were either gullible or experienced (recently treated with Interferon in addition to ribavirin in any event one year prior). All patients tried positive for serum HCV RNA genomic materials by SRT-PCR. The avoidance measures included, hepatic encephalopathy, pregnant females, hepatocellular carcinoma (HCC), and HBV or HIV coinfection. Patients with renal weakness (GFR under $30 \mathrm{ml} /$ minute), those with INR $>1.7$, serum egg whites $<2.8 \mathrm{~g} / \mathrm{dl}$, absolute bilirubin $>3 \mathrm{mg} / \mathrm{dl}$ or platelet tally $<50,000 / \mathrm{mm} 3$ were rejected. All patients were submitted to clinical assessment, research center testing for liver capacities, kidney capacities, and fasting glucose. Pregnancy test was accomplished for female patients in childbearing period. Stomach ultrasonography was performed to survey hepatic reverberation example of the liver, patency of gateway vein, presence of splenomegaly and to prohibit hepatocellular carcinoma.

\subsection{Medicine administration}

The anti-HCV drugs were administered according to guidelines regarding doses, routes of administration, and duration of therapy. The antiviral therapeutic regimens included Sofosbuvir at $400 \mathrm{mg}+$ Daclatasvir at $60 \mathrm{mg}+$ Ribavirin dosage was initiated at $600 \mathrm{mg}$ per day and gradually titrated upwards after exclusion of its significant side effects to reach 1000 . 


\subsection{Monitoring of treatment safety}

Patients were followed up every 4 weeks during treatment and 12 weeks post treatment (to test for SVR12) where history taking was done to document and manage any adverse event, and patients were asked specifically about the commonly reported adverse effects as headache, insomnia, fatigue, rash, dyspnea and G.I.T symptoms such as nausea,vomiting and diarrhea.

\subsection{Monitoring of treatment efficacy}

\section{Quantitative HCV-PCR was measured}

with a lower limit of detection of 15 IU prior to treatment, and 12 weeks after treatment where virologic response was considered when HCV RNA is less than lower limit of detection week 12 post treatment (SVR12) while treatment failure was defined as confirmed HCV RNA above LLOQ 12 weeks post treatment.

\subsection{Statistical Analysis}

Prospective follow up study will be conducted on patients diagnosed with hepatitis $\mathrm{C}$ infection (HCV RNA positive) \& will take Sofosbuvir\&Daclatasavir with or without Ribavirin as a dual or triple therapy. The recruited patients of the study who will be admitted to QenaFever,Gastro-enterology and Hepatology hospital between October 2016 to april 2017. Success of treatment was considered HCV RNA negative 12 weeks after the end of the treatment (SVR).

Categorical variables were described by number and percent $(\mathrm{N}, \%)$, where continuous variables described by mean and standard deviation (Mean, SD). Chi-square test was used to compare between categorical variables where continuous variables had been compared by t-test. Continuous variables were tested for normal distribution using Kolmogorove Smirnov test and Q-Q Plots. A two-tailed $\mathrm{p}<0.05$ was considered statistically significant. All analyses were performed with the IBM SPSS 24.0 software for WINDOS.

Data were tabulated, computerized and shown in the form of rate $(\%)$ and the standard deviation (SD). Chi-Square $\left(\chi^{2}\right)$ was used where appropriate. The Pvalue less than 0.05 were considered as statistically significant.

\section{Results}

Table (1) Baseline demographic data of studied groups.

\begin{tabular}{|c|c|c|c|c|c|}
\hline & & & \multicolumn{2}{|c|}{ Group age } & \multirow[t]{3}{*}{ P value } \\
\hline & & & $\begin{array}{l}\quad I \\
\text { Less than } 65 \\
\text { years }\end{array}$ & $\begin{array}{c}\text { II } \\
\text { more than } 65 \\
\text { years }\end{array}$ & \\
\hline & & & $n=100$ & $n=100$ & \\
\hline \multirow[t]{2}{*}{ Age } & & Mean & 45.6 & 67.4 & $<.05$ \\
\hline & & Std. Deviation & 9.16184 & 2.8885 & \\
\hline \multirow[t]{4}{*}{ Sex } & M & No. & 77 & 49 & $<.05$ \\
\hline & & & $77.0 \%$ & $49.0 \%$ & \\
\hline & $\mathrm{F}$ & No. & 23 & 51 & \\
\hline & & & $23.0 \%$ & $51.0 \%$ & \\
\hline \multirow[t]{2}{*}{ BMI } & & Mean & 27.077 & 27.899 & $>.05$ \\
\hline & & Std. Deviation & 4.56227 & 5.92184 & \\
\hline \multirow[t]{4}{*}{ Smoking } & no & No. & 73 & 80 & \\
\hline & & $\%$ & $73.0 \%$ & $80.0 \%$ & $>.05$ \\
\hline & yes & No. & 27 & 20 & \\
\hline & & $\%$ & $27.0 \%$ & $20.0 \%$ & \\
\hline \multirow{4}{*}{$\begin{array}{l}\text { Treatment } \\
\text { naiive }\end{array}$} & $\mathrm{E}$ & No. & 16 & 8 & \\
\hline & & $\%$ & $16.0 \%$ & $8.0 \%$ & $>.05$ \\
\hline & $\mathrm{N}$ & No. & 84 & 92 & \\
\hline & & $\%$ & $84.0 \%$ & $92.0 \%$ & \\
\hline
\end{tabular}

There was statistically significant difference as regards (Age and Sex) with mean age in group II (more than 65 years) is more than that in group I (less than 65 years) and males predominance in group I, females predominance in group II. There was no statistically significant difference as regards (BMI, smoking and treatment naiive).

Table (2) Sustained virological response S.V.R in group $\mathrm{I}(<65$ years) between group Ia (patients received dual therapy)and $\mathrm{Ib}$ (patients received triple therapy years.

\begin{tabular}{|c|c|c|c|c|c|}
\hline & & \multicolumn{2}{|c|}{ Group I } & \multirow[b]{2}{*}{$\begin{array}{c}\text { Total } \\
(n=100)\end{array}$} & \multirow[b]{2}{*}{$P$ value } \\
\hline & & $\frac{\text { I a SOF and DCL }}{(n=50)}$ & $\begin{array}{c}\text { I b SOF, DCL and } \\
\text { RBV } \\
(n=50) \\
\end{array}$ & & \\
\hline SVR & $\begin{array}{c}\text { Responder } \\
\text { Non responder }\end{array}$ & $\begin{array}{c}48 \\
2\end{array}$ & $\begin{array}{c}47 \\
3\end{array}$ & $\begin{array}{c}95 \\
5\end{array}$ & $>.05$ \\
\hline
\end{tabular}


This table shows that there was no significant difference in S.V.R finding between dual and triple therapy group in those patients less than 65 years as $\mathrm{P}$ value $>.05$.

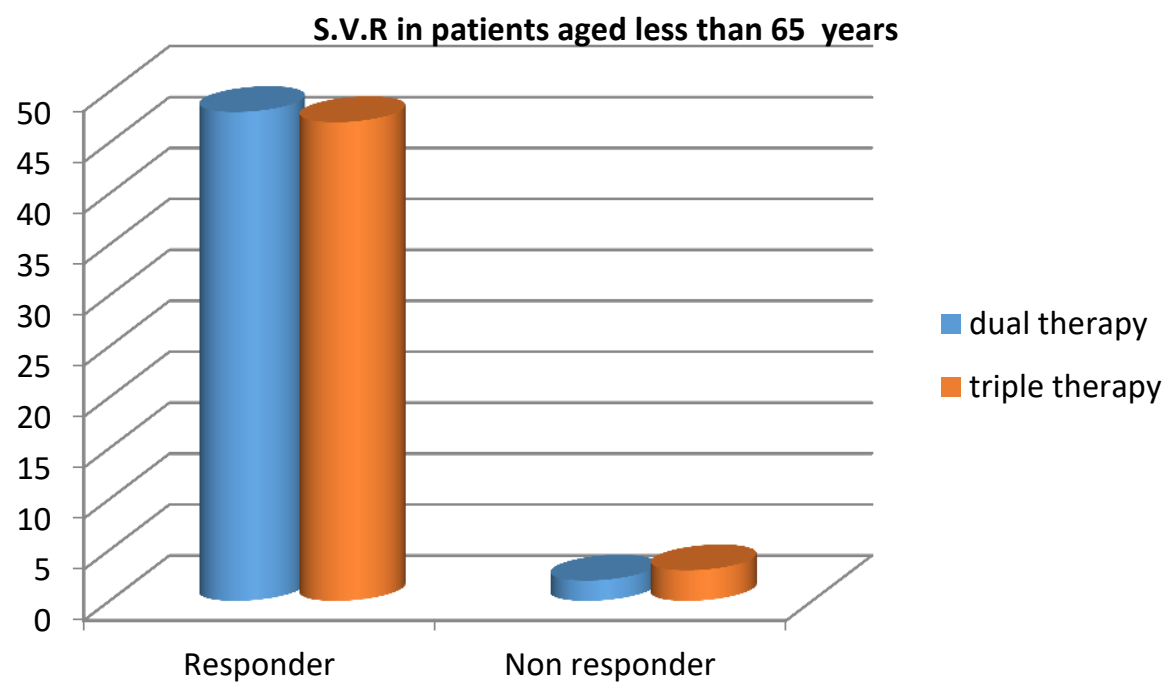

Fig (1) S.V.R in patients aged less than 65 years.

Table (3) Sustained virological response S.V.R in group II(> 65 years) between group IIa (patients received dual therapy)and IIb (patients received triple therapy years.

\begin{tabular}{|c|c|c|c|c|c|}
\hline & & \multicolumn{2}{|c|}{ Group II } & \multirow[b]{2}{*}{$\begin{array}{c}\text { Total } \\
(\mathbf{n}=\mathbf{1 0 0})\end{array}$} & \multirow[b]{2}{*}{$P$ value } \\
\hline & & $\begin{array}{c}\text { II a } \\
\text { SOF and DCL } \\
(n=50)\end{array}$ & $\begin{array}{c}\text { II b } \\
\text { SOF, DCL and RBV } \\
(n=50)\end{array}$ & & \\
\hline \multirow[t]{2}{*}{ SVR } & Responder & 46 & 47 & 93 & $>.05$ \\
\hline & Non responder & 4 & 3 & 7 & \\
\hline
\end{tabular}

This table shows that there was no significant difference in S.V.R finding between dual and triple in those more than 65 years as $\mathrm{P}$ value $>.05$.

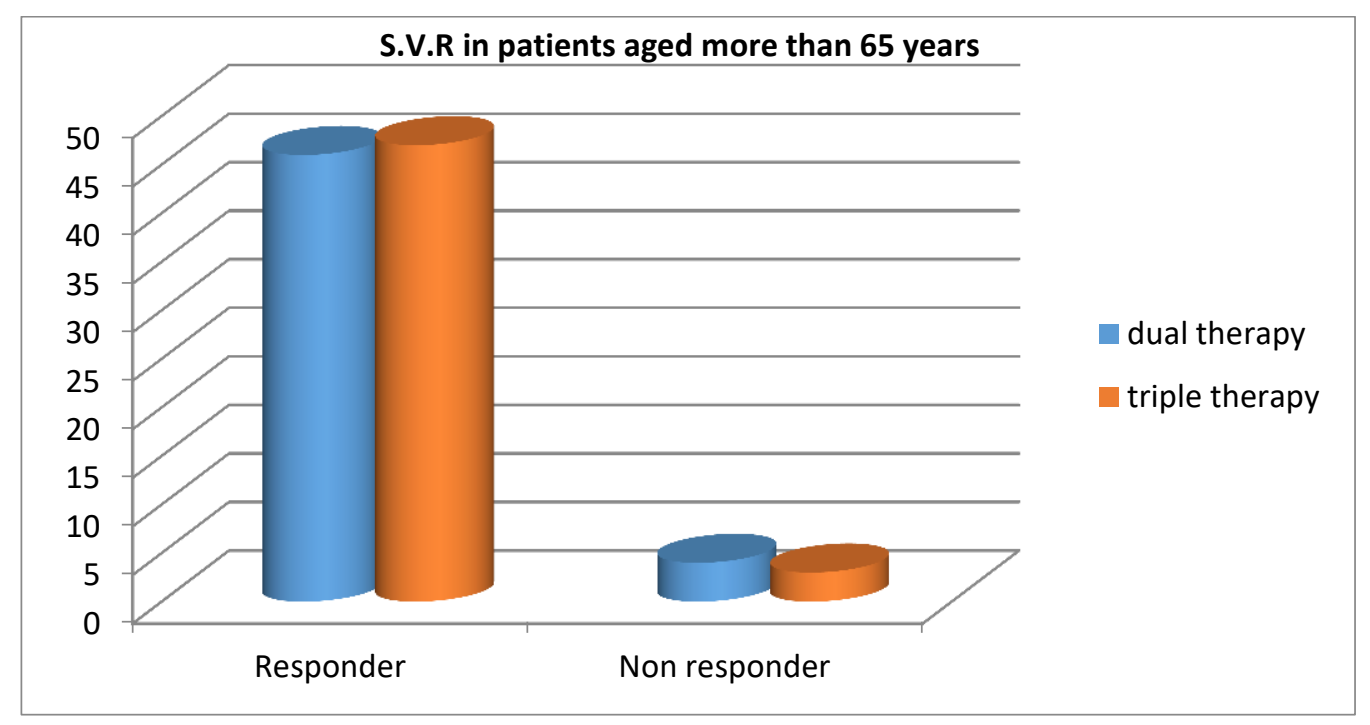

Fig (2) S.V.R in patients aged more than 65 years 
Table (4) Sustained virological response S.V.R in patients receive dual therapy(SOF+DCL)between group Ia( $<65$ years) and group $\mathrm{IIa}(>65$ years $)$.

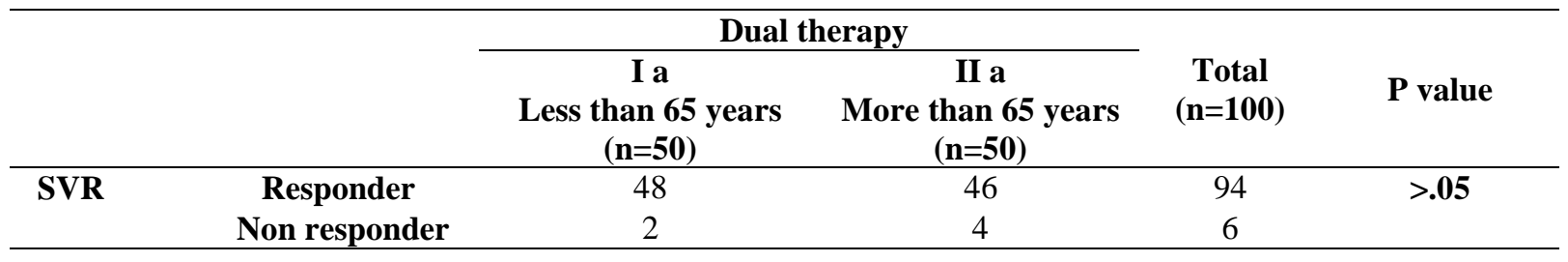

This table shows that there was no significant difference regarding S.V.R finding between patients <
65 years and > 65 years who received dual therapy (SOF and DCL) as P value $>.05$.

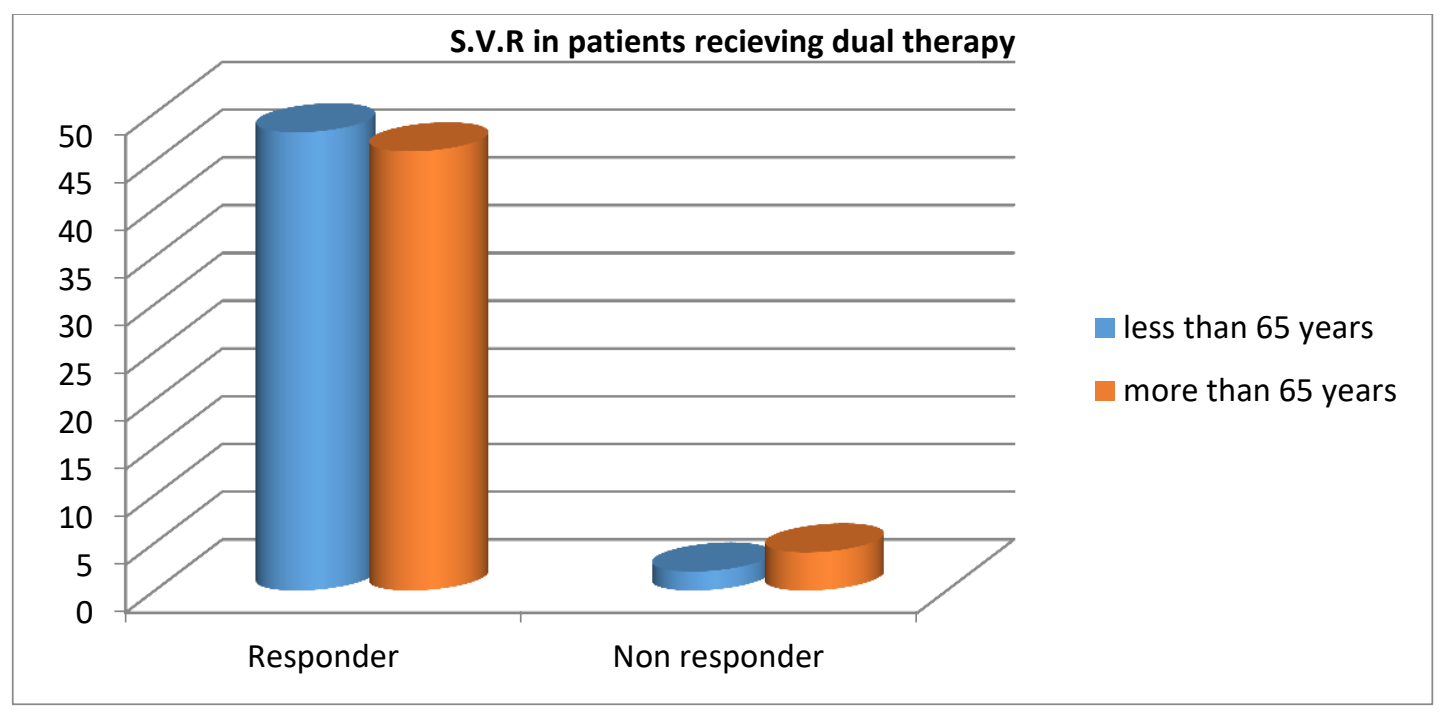

Fig (3) S.V.R in patients recieving dual therapy.

Table (5) Sustained virological response S.V.R in patients receive triple therapy (SOF + DCL +RBV) between group $\mathrm{Ib}(<65$ years $)$ and group $\mathrm{IIb}(>65$ years $)$.

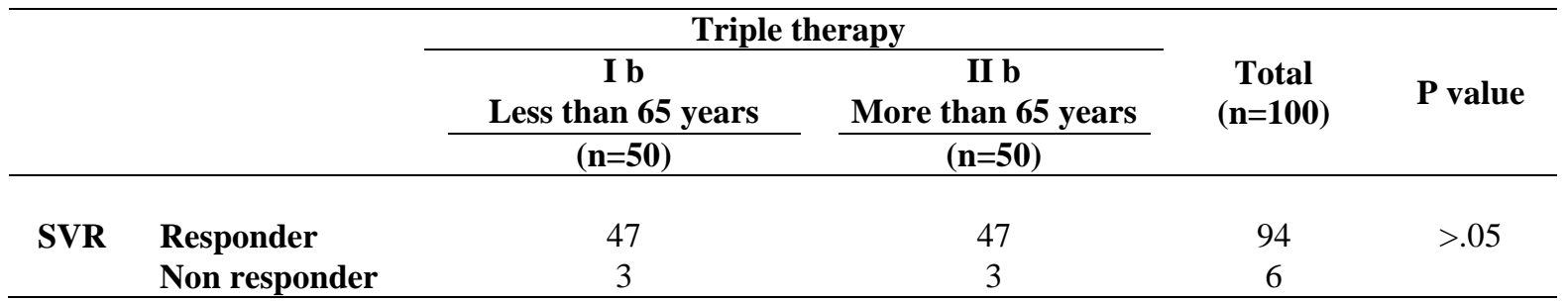

This table shows that there was no significant difference in S.V.R finding between patients $<65$ years and $>65$ years who received triple therapy $(\mathrm{SOF}, \mathrm{DCL}$ and $\mathrm{RBV}$ ) as $\mathrm{P}$ value $>.05$.

Table (6) Side effects reported during antiviral therapy in group $\mathrm{I}(<65$ years) between group Ia (patients received dual therapy) and $\mathrm{Ib}$ (patients received triple therapy) .

\begin{tabular}{lccc}
\hline \multirow{2}{*}{ Side effects } & \multicolumn{3}{c}{ Group I } \\
\cline { 2 - 3 } & $\begin{array}{c}\text { I a SOF and DCL (patients less } \\
\text { than 65 years) (n=50) }\end{array}$ & $\begin{array}{c}\text { I b SOF, DCL and RBV (patients less } \\
\text { than 65 years) }(\mathbf{n = 5 0})\end{array}$ & $\begin{array}{c}\text { P } \\
\text { value }\end{array}$ \\
\hline \multirow{2}{*}{ Abdominal pain } & 4 & & $<.05$ \\
Anaemia & 0 & 9 & $<.05$ \\
Arthralgia & 4 & 9 & $<.05$ \\
Diarrhea & 3 & 8 & $>.05$ \\
Fatigue & 3 & 10 & $<.05$ \\
\hline
\end{tabular}




\begin{tabular}{lllr}
\hline Table (6) Continue & & & \\
\hline Headache & 2 & 13 & $>.05$ \\
Insomnia & 5 & 14 & $<.05$ \\
Nausea & 5 & 13 & $<.05$ \\
Thrombocytopenia & 0 & 11 & $<.05$ \\
\hline
\end{tabular}

This table shows that there was statistically significant difference between group Ia (SOF and DCL less than 65 years) and $\mathrm{Ib}$ (SOF, DCL and RBV + less

On-treatment Safety and Tolerability than 65 years) $\mathrm{P}$ value $<.05$ as regards (Abdominal pain, Anaemia, Arthralgia, Fatigue , Insomnia, Nausea and Thrombocytopenia).

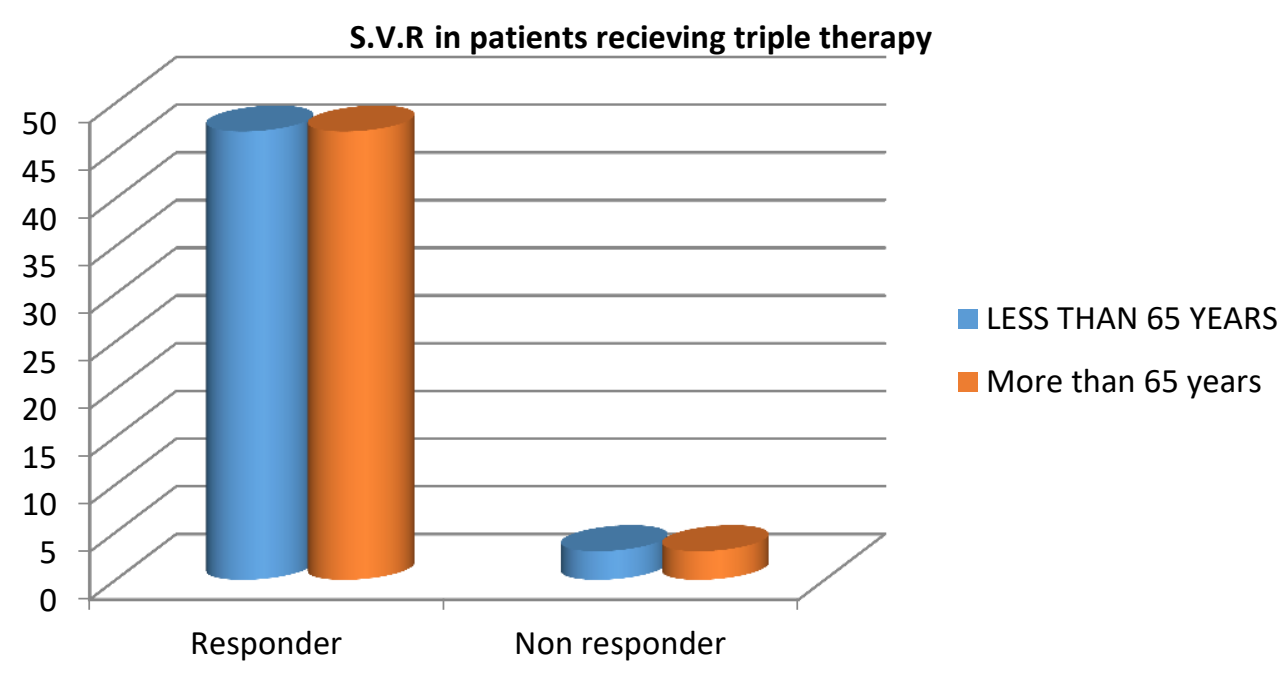

Fig (4) S.V.R in patients recieving triple therapy.

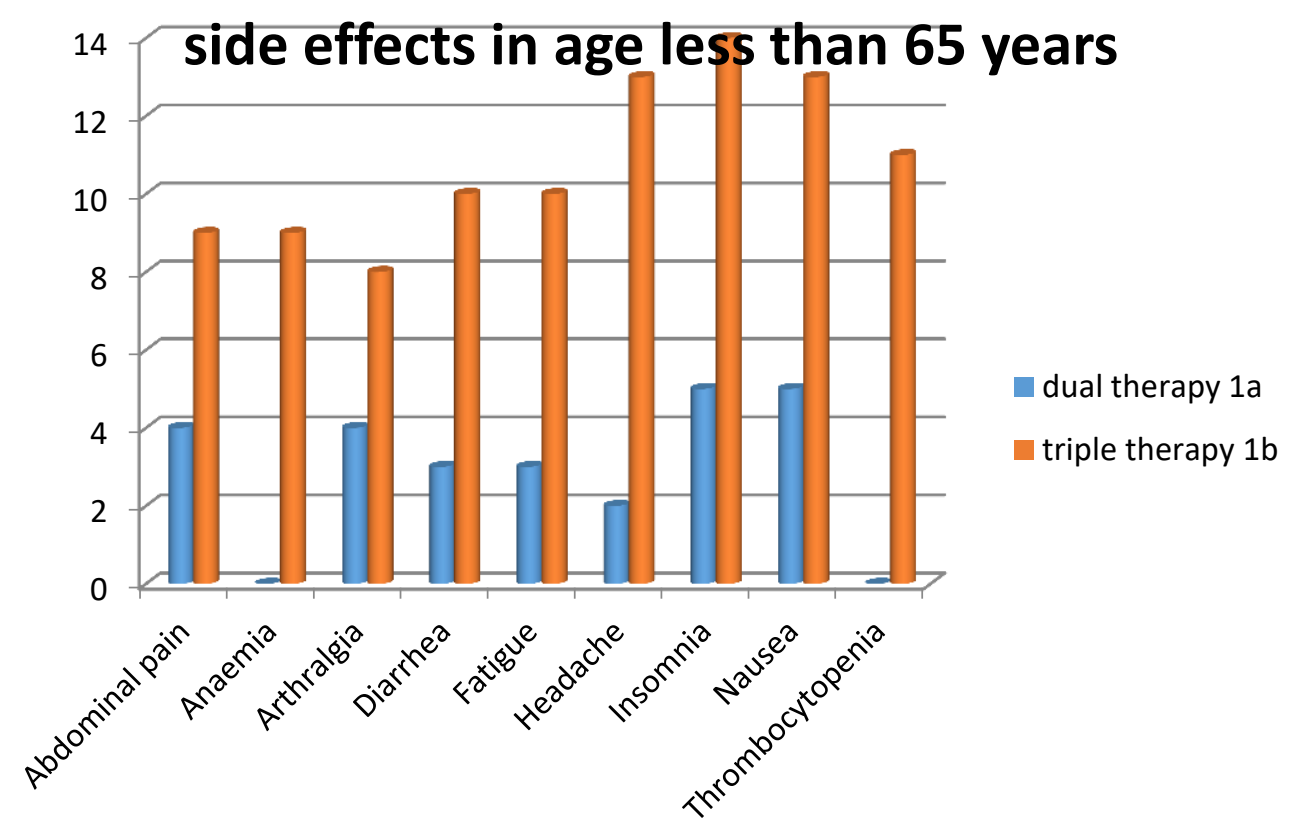

Fig (5) Side effects reported in patients younger than 65 years. 
Table (7) Side effects reported during antiviral therapy in group II( $>65$ years) between groupIIa (patients received dual therapy) andIIb (patients received triple therapy).

\begin{tabular}{lccc}
\hline & \multicolumn{2}{c}{ Group II } \\
\cline { 2 - 4 } Side effects & $\begin{array}{c}\text { II a } \\
\text { SOF and DCL (patients } \\
\text { more than 65 years) }(\mathbf{n = 5 0})\end{array}$ & $\begin{array}{c}\text { II b } \\
\text { SOF, DCL and RB V (patients more } \\
\text { than 65 years) }(\mathbf{n = 5 0})\end{array}$ & $\begin{array}{c}\text { P } \\
\text { value }\end{array}$ \\
\hline Abdominal pain & & & \\
Anaemia & 8 & 15 & $>.05$ \\
Arthralgia & 2 & 16 & $<.05$ \\
Diarrhea & 6 & 15 & $>.05$ \\
Fatigue & 7 & 15 & $>.05$ \\
Headache & 9 & 16 & $<.05$ \\
Insomnia & 7 & 15 & $<.05$ \\
Nausea & 7 & 16 & $>.05$ \\
Thrombocytopenia & 7 & 16 & $<.05$ \\
\hline
\end{tabular}

This table shows that there were statistically significant differences between group IIa (SOF and $\mathrm{DCL}+$ more than 65 years) and II b (SOF, DCL and
$\mathrm{RBV}+$ more than 65 years) $\mathrm{P}$ value $<.05$ as regards (anaemia, fatigue, headache, nausea and thrombocytopenia).

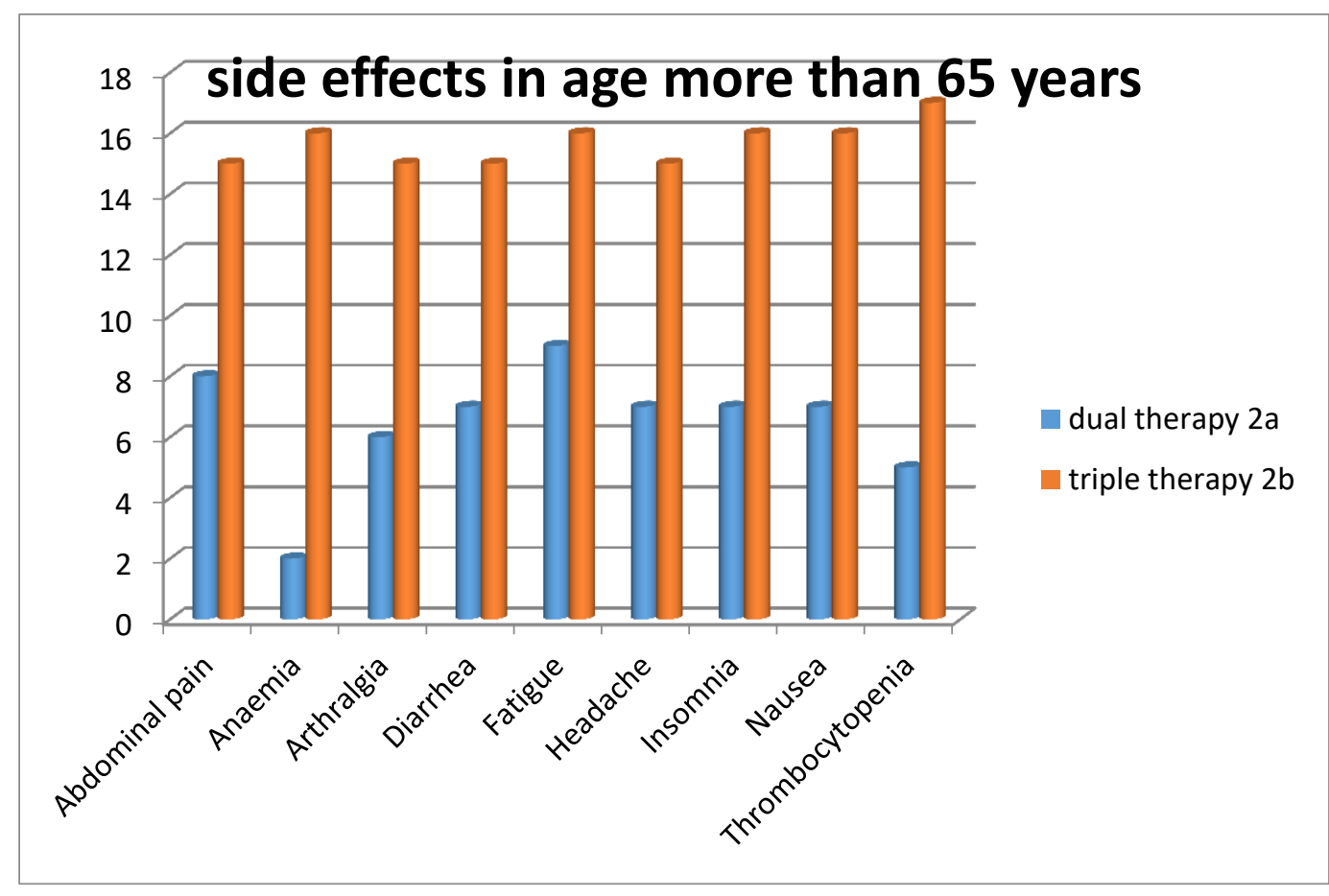

Fig (6) Side effects reported in patients older than 65 year.

Table (8) Side effects reported during antiviral therapy in patients receive dual therapy(SOF+DCL) between group Ia $(<65$ years) and IIa $(>65$ years $)$.

\begin{tabular}{lccc}
\hline & \multicolumn{2}{c}{ Dual therapy } & P value \\
\cline { 2 - 3 } Side effects & $\begin{array}{c}\text { I a } \\
\text { SOF and DCL (patients Less } \\
\text { than years 65 years) }\end{array}$ & $\begin{array}{c}\text { II a } \mathbf{5 0} \text { ) } \\
\text { SOF and DCL (patients } \\
\text { more than 65 years) (n=50) }\end{array}$ \\
\hline Abdominal pain & 4 & 8 & $>.05$ \\
Anaemia & 0 & 2 & $>.05$ \\
Arthralgia & 4 & 6 & $<.05$ \\
Diarrhea & 3 & 7 & $>.05$ \\
Fatigue & 3 & 9 & $>.05$
\end{tabular}


Table (8) Continue

\begin{tabular}{llll}
\hline Headache & 2 & 7 & $<.05$ \\
Insomnia & 5 & 7 & $<.05$ \\
Nausea & 5 & 7 & $>.05$ \\
Thrombocytopenia & 0 & 5 & $>.05$ \\
\hline
\end{tabular}

This table shows that there was statistically significant differences between group Ia (SOF and
DCL + less than 65 years) and IIa (SOF and DCL + more than 65 years) $\mathrm{P}$ value $<.05$ as regards (arthralgia, headache and insomnia).

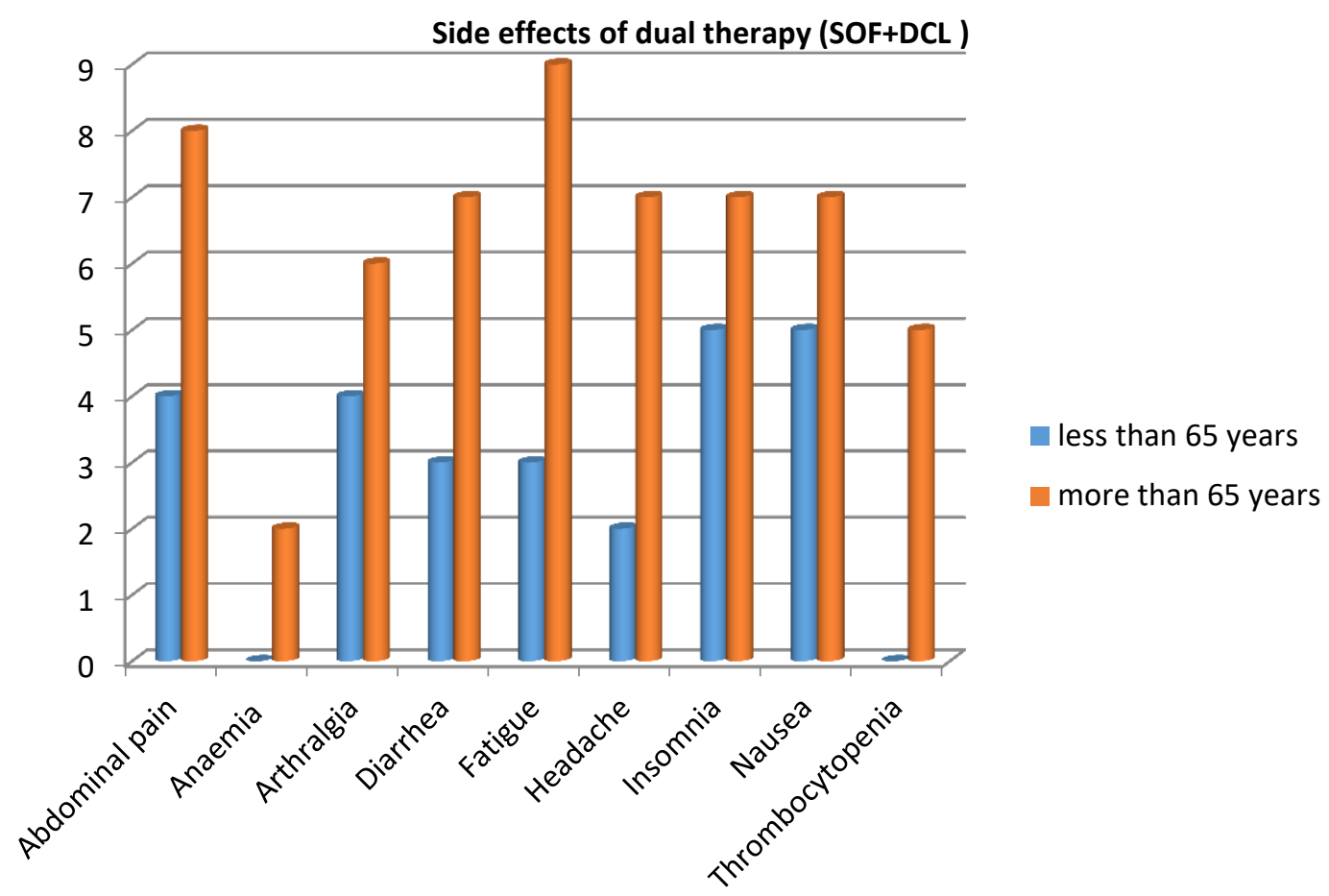

Fig (7) Side effects of dual therapy (SOF+DCL ).

Table (9) Side effects reported during antiviral therapy in patients receive triple therapy(SOF+DCL+RBV) between group Ib ( $<65$ years) and IIb ( $>65$ years).

\begin{tabular}{lccc}
\hline \multirow{2}{*}{ Side effects } & \multicolumn{2}{c}{ Triple therapy } \\
\cline { 2 - 4 } & $\begin{array}{c}\text { I bSOF, DCL and RBV (patients less } \\
\text { than 65 years) }\end{array}$ & $\begin{array}{c}\text { II b SOF, DCL and RBV } \\
\text { (patients more than 65 years) } \\
(\mathbf{n = 5 0})\end{array}$ & $\begin{array}{c}\text { P } \\
\text { value }\end{array}$ \\
\hline Abdominal pain & 9 & 15 & $>.05$ \\
Anaemia & 9 & 16 & $>.05$ \\
Arthralgia & 8 & 15 & $>.05$ \\
Diarrhea & 10 & 15 & $>.05$ \\
Fatigue & 10 & 16 & $>.05$ \\
Headache & 13 & 15 & $>.05$ \\
Insomnia & 14 & 16 & $>.05$ \\
Nausea & 13 & 16 & $>.05$ \\
Thrombocytopenia & 11 & 17 & $>.05$ \\
\hline
\end{tabular}

This table shows that there were no statistically significant differences between group $\mathrm{Ib}$ (SOF, DCL and RBV + less than 65 years) and II b (SOF, DCL and $\mathrm{RBV}+$ more than 65 years) regarding the reported side effects. 


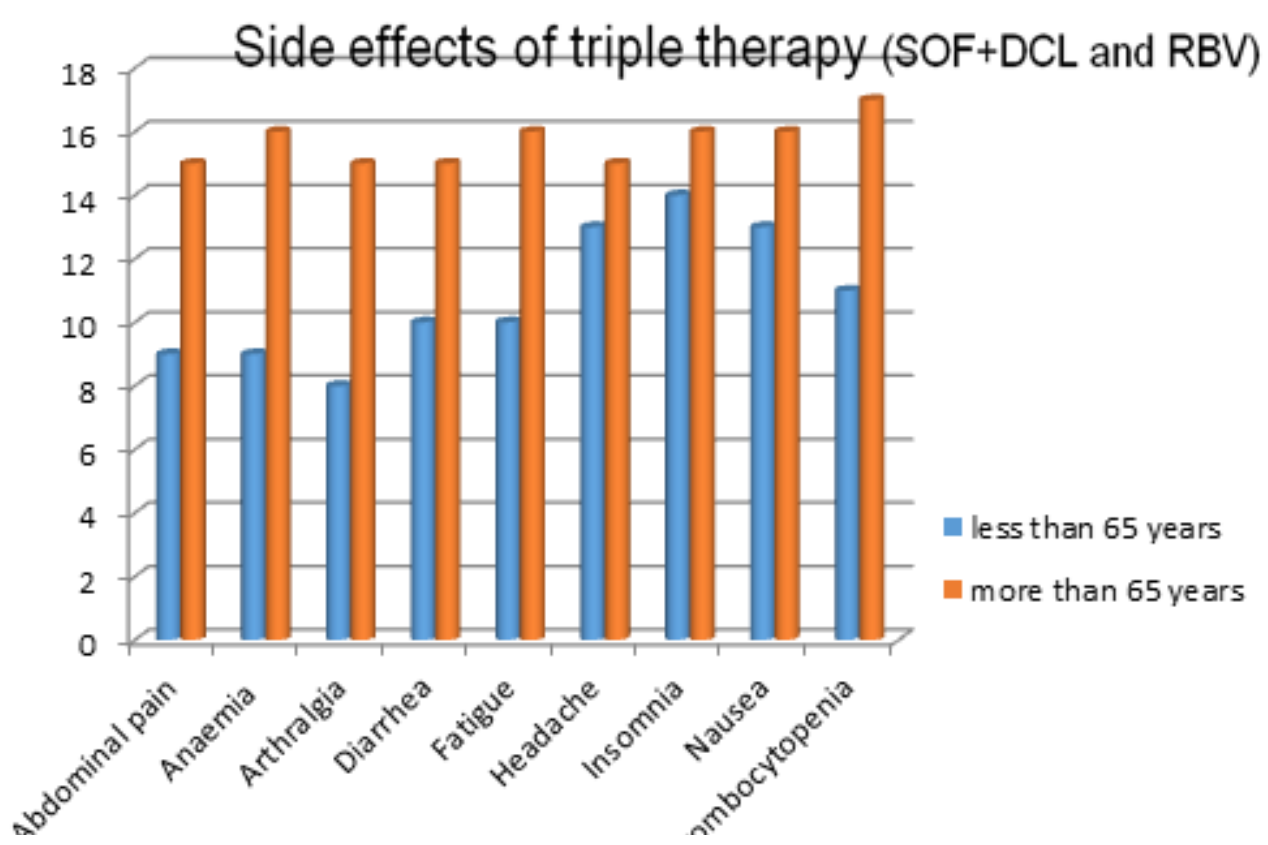

Fig (8) Side effects f triple therapy(SOF+DCL+RBV).

\section{Discussion}

In the current examination, there was 77 guys and 23 females in chronic H.C.V group. Aged below 65 years and 49 guys and 51 females in bunch ongoing H.C.V.aged above 65 years. There is critical distinction in Mean age contrast in cases $<65$ years and $>65$ years (45.5 and 67.4 separately) $\mathrm{P}$ esteem $=.000$.

In past examinations as omar and his colleges (2018) 18378 patients were tried out his investigation with mean age $49.4 \pm 11.6$ years [21].

What's more, among complete of 2293 subjects who enrolled in Saab et al., study, 2029 (88\%) were $<65$ years old with mean 5.2 and 264 were $>65$ years old with mean 6.8 [25].

In the current investigation, $73 \%$ of cases under 65 years were nonsmokers in contrast with $80 \%$ of cases $>65$ years. There was no measurably noteworthy distinction as respects smoking.

In the current examination, there was no factually huge contrast as respects BMI

In the current investigation, there was factually noteworthy distinction as respects Sex. In the current investigation, there was 77 guys and 23 females in bunch persistent H.C.V. Under 65 years and 49 guys and 51 females in bunch persistent H.C.V. over 65 years.

2293 subjects were taken on Saab et al., study, of whom 2029 (88\%) with male power were <65 years old and 264 with female transcendence were $>65$ years old [25].

Additionally, 18378 patients in Omar et al., study have female transcendence who got treatment with SOF-DCV or SOF-DCV-RBV.(57.6\%) [21].

Conversely, Tarao et al., analyzed the viability and wellbeing of double treatment in A sum of 197 patients, and included 56 old patients and 141 non-old patients. The male/female proportion was almost the equivalent in the two gatherings $(\mathrm{P}=0.719)$ [27].

In the current investigation, 16 cases were experienced patients and 84 cases were naiive patients under 65 years while 8 cases with treatment and 92 cases taking naiive treatment over 65 years, with no measurably noteworthy contrast as respects this point.

10025 patients were remembered for concentrate out of which $42(33.6 \%)$ were male and $83(66.4 \%)$ were female. Mean age was $47.06+10.8$ years. Gotten SOF and DCL, the continued virological reaction (SVR) was accomplished by $123(98.4 \%$ ) and 124 (99.2\%) patients individually [6].

In the current investigation, there was no critical distinction in S.V.R finding between bunch Ia (SOF and DCL) and Ib (SOF, DCL and RBV) in those under 65 years as $\mathrm{P}$ esteem $>.05$.

C.H. Liu [16] shows the SVR12 paces of SOFbased DAA regimens. The SVR12 rates were practically identical concerning age utilization of RBV.

The expansion of RBV to the $\mathrm{SOF}+\mathrm{DAC}$ routine expanded SVR rates to 83 and $89 \%$, separately, without huge contrast [14].

Also, Babatin et al, uncovered that SOF/DCV with or without RBV was exceptionally viable in $\mathrm{HCV}$ treatment by accomplishing SVR12 100\% in without critical distinction [4].

An open-mark observational examination by Shiha et al., that portrays the impact of multi week of day by day oral Sofosbuvir (SOF) in addition to Daclatasvir (DCV) with or without ribavirin (RBV) in the Egyptian Liver Research Institute and Hospital, Mansoura, Egypt. They discovered supported viral reaction following 12 weeks of end of treatment (SVR12) was accomplished in $96.6 \%$ of the patients accepting 12 weeks of DCV/SOF treatment, in $95.7 \%$ of the patients getting 12 weeks of DCV/SOF/RBV [26]. 
Likewise, an enormous partner of 946 patients $<65$ years with constant $\mathrm{HCV}$ was tried out examination by Abdel-Moneim et al., Patients were ordered into: bunch 1 was treated with $\mathrm{SOF} / \mathrm{DCV}$ and gathering 2 was treated with SOF/DCV/RBV. They found as concerning viral reaction, SVR12 rate was accomplished by $94 \%$ in the general patients (891/946); by $95 \%$ (718/758) in SOF/DCV gathering, and by $92 \%(173 / 188)$ in SOF/DCV/RBV gathering [1].

In the current examination, there was no noteworthy contrast in S.V.R finding between bunch IIa (SOF and DCL). What's more, IIb (SOF, DCL and $\mathrm{RBV})$ in those over 65 years as $\mathrm{P}$ esteem $>.05$.

Sixteen percent of patients treated with $2 \mathrm{D}$ - based by Villani, $26 \%$ of sof-based treatment demonstrated imperceptible HCV-RNA by about a month of treatment, all patients accomplished SVR12 (99.3\% in old) [29].

In patients $>65$ years were enlisted to get openname daclatasvir $60 \mathrm{mg}+$ sofos-buvir $400 \mathrm{mg}$ once every day for 12 weeks. SVR12 was accomplished in $90.1 \%$ and $86.3 \%$ of treatment-innocent and treatmentexperienced patients, individually [19].

In the current investigation, there was no huge contrast in S.V.R finding between bunch Ia (Less than 65 years) and IIa (More than 65 years)in those taking double treatment (SOF and DCL) as P esteem >.05.

Welzel et al.(30) found that SVR12 rates were (91\% with DCV+SOF; $88 \%$ with DCV+SOF+RBV) [30].

Sixteen percent of patients treated with 2D - based by Villani, $26 \%$ of sof-based treatment indicated imperceptible HCV-RNA by about a month of treatment, all patients accomplished SVR12 (98.3\% in more youthful patients) [29].

In the current investigation, there was no noteworthy contrast in S.V.R finding between bunch Ib (Less than 65 years) and IIb (More than 65 years) in those taking triple treatment (SOF, DCL and RBV) as $\mathrm{P}$ esteem $>.05$.

Likewise, in study, the proportion of patients accomplishing SVR after double treatment was almost the equivalent in the two gatherings. As of the older patients, $51(91.1 \%)$ accomplished SVR while in the non-old patients, 127 (90.1\%) accomplished SVR [27].

As per our finding, the viability results of the current investigation were in corresponding with consequences of El-Khayat et al [9]. All patients under 65 years got SOF and DCV every day notwithstanding weight-based ribavirin (RBV) for 12 weeks and. They discovered huge distinction in Sustained virological reaction at 12 weeks after finish of treatment (SVR12) rate was $92 \%$ in credulous cirrhotic patients and $87 \%$ in past treated patients [9].

Leroy et al., assessed DCV/SOF in addition to ribavirin $(\mathrm{RBV})$ in patients in two gathering either $>65$ or $<65$ years with repaid cirrhosis for 12 weeks. SVR12 in patients with cirrhosis, SVR12 was $83 \%$ [14].
In the current examination, correlation among responder and non-responder in bunch Ia double treatment (SOF and DCL+ under long term). No critical contrast among responders and non-responders in discoveries in the gathering taking double treatment (SOF, DCL) and under 65 years.

10025 patients were remembered for Butt and Shah, concentrate out of patient got SOF, DCL with Mean age was $47.06+10.8$ years. $102(81.6 \%)$ patients were treatment innocent, $7(5.6 \%)$ treatment nonresponders and $16(12.8 \%)$ were relapsers [6].

In the current investigation, examination among responder and non-responder in bunch IIa double treatment (SOF and DCL+ over long term). No huge contrast among responders and non-responders in discoveries in the gathering taking double treatment or more 65 years.

In general, in Vermehren et al., investigation of patients matured 65 years and more seasoned got (daclatasvir/sofosbuvir \pm ribavirin and daclatasvir/sofosbuvir tribavirin).There was no virological treatment disappointment in patients who got in any event $80 \%$ of the proposed treatment term and the SVR rate in these patients was $100 \%(\mathrm{n}=$ 134/134) [16].

In the current investigation, examination among responder and non-responder in bunch $\mathrm{Ib}$ triple treatment (SOF, DCL and RBV under long term). No noteworthy distinction among responders and nonresponders in discoveries in the gathering taking triple treatment and under 65 years.

Another huge investigation in Egypt by Omar et al., on persistent $\mathrm{HCV}$ patients who were treated for 12 weeks with DCV in addition to $\mathrm{SOF} \pm$ ribavirin (RBV) inside the public hepatitis $\mathrm{C}$ treatment program in Egypt. Their outcomes indicated that, among 18378 patients with HCV began treatment with SOF-DCV with or without RBV, by and large, $95.1 \%$ accomplished response $(95.4 \%$ among patients treated without RBV and $94.7 \%$ for patients treated with RBV, $\mathrm{P}=0.32$ ) [21].

Supported virological reaction pace of $92 \%$ Twenty five gullible patients (6\%); were non responders $<65$ years got SOF in addition to daclatasvir with or without ribavirin [14].

106 G3 cirrhotic patients were tried out examination by Lionetti et al., (70.8\% guys, mean age $55.3 \pm 7.6$ years) got ourse of sofosbuvir in addition to daclatasvir \pm ribavirin All patients finished 12-week subsequent post-treatment, and 104 (98.1\%) acquired supported virological reaction $(100 \%$ in ribavirin treated patients versus $90.4 \%$ without ribavirin; $\mathrm{p}=0.04)$ [15].

In the current investigation, examination among responder and non-responder in bunch IIb triple treatment (SOF, DCL and RBV under long term). No critical distinction among responders and nonresponders in discoveries in the gathering taking triple treatment or more 65 years. 
Generally, in Welzel et al., study, SVR12 was accomplished by $91 \%$ of the 460 patients in the essential examination (adjusted goal to-treat (mITT)), including $92 \%$ of patients treated with $\mathrm{DCV}+\mathrm{SOF}$ and $89 \%$ of those treated with DCV+SOF+RBV. Reaction rates were higher (97\% and 96\%, individually) [30].

Altogether, 409 patients mono-contaminated with HCV selected Pol et al., study were given sofosbuvir + daclatasvir without ribavirin $(n=318)$ or with ribavirin $(n=91)$. SVR12 rates were $84.9 \%$ following 12 weeks of treatment with sofosbuvir and daclatasvir alone. With the expansion of ribavirin, SVR4 rates were $100 \%$ with 12 weeks [23].

Additionally, Coilly et al. shown that the mix of DCV/SOF with or without RBV for 12 or 24 weeks empowered $96 \%$ SVR12 rate [7].

In the current investigation, symptoms of under 65 years of both double and triple treatment bunch incorporate stomach torment (13 cases), paleness (9 cases), weariness (13 cases) sickness (18 cases), thrombocytopenia(11

cases), arthralgia(12), diarrhea(13), headache (15) and insomnia [19].

As indicated by Vermehren et al., study, results were commonly gentle, they watched the most widely recognized result were exhaustion (35\%), dyspnoea $(11 \%)$, cerebral pain $(22 \%)$, pruritus $(7 \%)$ rash $(7 \%$ and a sleeping disorder $(6 \%$ and $7 \%)$ in patients $<65$. [16].

In the current investigation, results of over 65 years of both double and triple treatment bunch incorporate abd

\section{Conclusion}

From this study, we found that both Triple and Dual therapy achevied high rates of sustained virologic response in Egyptian patients with chronic HCV, with high safety and tolerability profile.

\section{Reference}

[1] A. Abdel-Moneim, A.Aboud, M.Abdel-Gabaar. Efficacy and safety of sofosbuvir plus daclatasvir with or without ribavirin: large real-life results of patients with chronic hepatitis C genotype 4 . Hepatology international, Vol.8,PP.1-8,2018.

[2] O.A.Ahmed, E.Safwat M.O. Khalifa. Sofosbuvir Plus Daclatasvir in Treatment of Chronic Hepatitis C Genotype 4 Infection in a Cohort of Egyptian Patients: An Experiment the Size of Egyptian Village. International J.,hepatology ,2018.

[3] S.Alonso, M.Riveiro-Barciela, I.Fernandez. Effectiveness and safety of sofosbuvir-based regimens plus an NS 5A inhibitor for patients with HCV genotype 3 infection and cirrhosis. Results of a multicenter real-life cohort. J.,viral hepatitis, Vol.24,PP. 304-311,2017.

[4] M.A.Babatin, A.S.Alghamdi, A.Albenmousa. Efficacy and safety of simeprevir or daclatasvir in combination with sofosbuvir for the treatment of hepatitis C genotype 4 infection. J.,clinical gastroenterology, Vol. 52,PP.452-457,2018.

[5] L.Boglione, S.M.Pinna, C.S.Cardellino. Treatment with daclatasvir and sofosbuvir for 24 weeks without ribavirin in cirrhotic patients who failed first-generation protease inhibitors. Infection , Vol.45,PP.103-106,2017.

[6] Z.Butt , S.M.A.Shah. Daclatasvir plus Sofosbuvir with or without ribavirin in patients with chronic Hepatitis $\mathrm{C}$ genotype $3 \mathrm{a}$ in Pakistani population A real world experience. Pak J Med Sci, Vol. 35,PP.409-413,2019.

[7] A.Coilly, C.Fougerou-Leurent, V.Ledinghen. Multicentre experience using daclatasvir and sofosbuvir to treat hepatitis $\mathrm{C}$ recurrence-The ANRS CUPILT study. J.,hepatology, Vol. 65,PP. 711-718,2016.

[8] Doss W,Shiha G,Hassany M. 2015 Sofosbuvir plus ribavirin for treating Egyptian patients with hepatitis C genotype.J., Hepatology Vol. 63 j 581585.

[9] H.El-Khayat, Y.Fouad, H.0.Mohamed . Sofosbuvir plus daclatasvir with or without ribavirin in 551 patients with hepatitis C-related cirrhosis, genotype 4. Alimentary pharmacology \& therapeutics, Vol. 47,PP. 674-679,2018.

[10] 10- A.Elsharkawy, M.Hashem, R.Fouad. Safety and efficacy of the generic products of sofosbuvir and daclatasvir in treatment of HCV genotype 4 Egyptian patients Merit Research J.,Medicine and Medical Sciences, Vol. 5(4) PP. 209-213,2017.

[11]X.Forns, S.C.Gordon, E.Zuckerman. Grazoprevir and elbasvir plus ribavirin for chronic $\mathrm{HCV}$ genotype-1 infection after failure of combination therapy containing a direct-acting antiviral agent. J.,hepatology, Vol. 63,PP.564-572,2015.

[12] E.Hathorn , A.M.ElsharkawyManagement of hepatitis $\mathrm{C}$ genotype 4 in the directly acting antivirals era . BMJ Open Gastro, Vol.8,PP.3:e000112,2016.

[13] K.Lacombe, H.Fontaine, C.Dhiver. Real-world efficacy of daclatasvir and sofosbuvir, with and without ribavirin, in $\mathrm{HIV} / \mathrm{HCV}$ coinfected patients with advanced liver disease in a French early access cohort. J.,acquired immune deficiency syndromes ,Vol. 75, PP. 97,2017

[14] V. Leroy, P. Angus, J.P. Bronowicki ,Daclatasvir, sofosbuvir, and ribavirin for hepatitis $\mathrm{C}$ virus genotype 3 and advanced liver disease: a randomized phase III study (ALLY-3+): Hepatology ,Vol. 63,PP. 1430-1441,2016

[15]R. Lionetti, P. Piccolo, I .Lenci, Daclatasvir, sofosbuvir with or without ribavirin for 24 weeks in hepatitis $\mathrm{C}$ genotype 3 cirrhosis: A real-life study. Annals of hepatology,Vol. 18,PP.434438,2019

[16]C.H. Liu, Y.J. Huang, S.S. Yang, Generic sofosbuvir-based -free direct acting antiviral agents for patients with chronic hepatitis $\mathrm{C}$ virus 
infection: a real-world multicenter observational study. Scientific reports, Vol.25(5),PP. 8,2018

[17] S. Manolakopoulosa, G. Zacharakisb , M. Zissisd , Safety and effi cacy of daclatasvir in the management of patients with chronic hepatitis $\mathrm{C}$. Annals of Gastroenterology , Vol.29, PP.282296,2016.

[18] H. M.Marleen , F. Adam , New Drug Mechanism Sofosbuvir and daclatasvir. Br J Clin Pharmacol ,Vol. 82, PP. 878-879 878, 2016.

[19] D.R. Nelson, J.N.Cooper, J.P.Lalezari ,All-oral 12 week treatment with daclatasvir plus sofosbuvir in patients with hepatitis $\mathrm{C}$ virus genotype 3 infection: ALLY-3 phase III study. Hepatology,Vol.61(4), PP.1127-1135,2015.

[20] E. Ogawa, N. Furusyo, H.Nomura ,Effectiveness and safety of sofosbuvir plus ribavirin for $\mathrm{HCV}$ genotype 2 patients 65 and over with or without cirrhosis. Antiviral research ,Vol. 136,PP. 3744,2016

[21]H. Omar, W. El Akel, T.Elbaz , Generic daclatasvir plus sofosbuvir, with or without ribavirin, in treatment of chronic hepatitis C: realworld results from 18378 patients in Egypt . Alimentary pharmacology \& therapeutics ,Vol. 47, PP. 421-431,2018.

[22] M. Omata, S. Nishiguchi, Y.Ueno, Sofosbuvir plus ribavirin in Japanese patients with chronic genotype 2 HCV infection: an open-label, phase 3 trial. J.,viral hepatitis, Vol.21,PP. 762-768,2014.

[23] S. Pol, M. Bourliere, S. Lucier, Safety and efficacy of the combination daclatasvir-sofosbuvir in HCV genotype 1-mono-infected patients from the French observational cohort ANRS CO22 HEPATHER. J hepatol ,Vol.62,PP. S258,2015.

[24]F. Poordad, E.R. Schiff, J.M. Vierling, Daclatasvir with sofosbuvir and ribavirin for hepatitis $C$ virus infection with advanced cirrhosis or post-liver transplantation recurrence. Hepatology. May, Vol.63(5), PP.1493-505,2016.

[25] S. Saab, S.H.Park, M. Mizokami, alSafety and efficacy of ledipasvir/sofosbuvir for the treatment of genotype 1 hepatitis $C$ in subjects aged 65 years or older. Hepatology ,Vol. 63 , PP.1119$1112,2016$.

[26] G. Shiha, R. Soliman, M.ElBasiony ,Sofosbuvir plus Daclatasvir with or without ribavirin for treatment of chronic HCV genotype 4 patients: real-life experience. Hepatology international, Vol. 60,PP. 1-9,2018.

[27] K. Tarao, K. Tanaka, A. Nozaki, Efficacy and safety of dual therapy with daclatasvir and asunaprevir in elderly patients. World J Hepatol ,Vol.9,PP.544-550,2017.

[28] J. Vermehren, K.H. Peiffer, C. Welsch ,The efficacy and safety of direct acting antiviral treatment and clinical significance of drug-drug interactions in elderly patients with chronic hepatitis $\mathrm{C}$ virus infection. Alimentary pharmacology \& therapeutics,Vol. 44, PP.856$865,2016$.

[29] R.Villani, I. Donatiello, F.Barone, Efficacy and safety of direct-acting antivirals in elderly with chronic hepatitis $\mathrm{C}$ : results from a retrospective cohort study. Official J.,the Italian Society of Gerontology and Geriatrics , Vol. 66,PP.4655,2018

[30] T.M. Welzel, J .Petersen, K. Herzer, Daclatasvir plus sofosbuvir, with or without ribavirin, achieved high sustained virological response rates in patients with $\mathrm{HCV}$ infection and advanced liver disease in a real-world cohort. Gut: gutjnl ,Vol.31,PP.24-44,2016. 